\title{
Drawing Venn Diagrams
}

\author{
Frank Ruskey \\ University of Victoria, Victoria, B.C. V8W 3P6, Canada, \\ fruskey@cs.uvic.ca
}

\begin{abstract}
Venn diagrams have long been used as an aid in understanding set containment relationships and in certain logical arguments. They also have interesting combinatorial properties. This talk is a survey some of what is known about Venn diagrams, particularly the aesthetic and extremal properties of the drawings, including recent results about symmetric Venn diagrams. Connections with graph drawing, of which there are many, will be emphasized. For more about Venn diagrams see http://www.theory.cs.uvic.ca/ ${ }^{\sim} \cos / v e n n$.
\end{abstract}
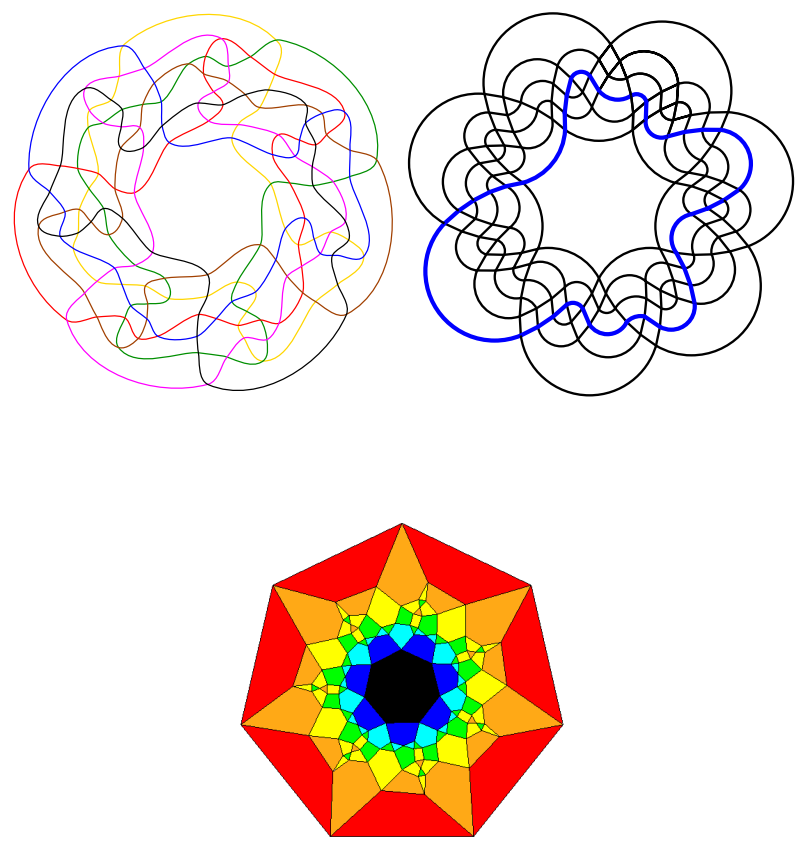